\title{
Counting Dislocations in Microcrystals by Coherent X-Ray Diffraction
}

\author{
V. L. R. Jacques, ${ }^{1, *}$ D. Carbone, ${ }^{1}$ R. Ghisleni, ${ }^{2}$ and L. Thilly ${ }^{3}$ \\ ${ }^{1}$ European Synchrotron Radiation Facility, 6 rue Jules Horowitz, BP220, 38043 Grenoble Cedex, France \\ ${ }^{2}$ Laboratory for Mechanics of Materials and Nanostructures, EMPA, Swiss Federal Laboratories for Materials Testing and Research, \\ Feuerwerkerstrasse 39, CH-3602 Thun, Switzerland \\ ${ }^{3}$ Institut Pprime, CNRS-University of Poitiers-ENSMA, SP2MI, 86962 Futuroscope, France \\ (Received 8 May 2013; revised manuscript received 26 June 2013; published 7 August 2013)
}

\begin{abstract}
We present here an unprecedented way of quantifying the number of dislocations in microcrystals. This method relies on a combination of several state-of-the-art techniques: coherent x-ray diffraction used as a local probe, together with the controlled compression of micro-objects. We demonstrate that by using this method, dislocations in the microcrystal can be detected and their number precisely quantified. This cannot be done with other techniques in a nondestructive way. Our method opens a route for the study of many small-scale systems with defect-dependent physical properties and it could become a critical tool for addressing future challenges in nanotechnology.
\end{abstract}

DOI: 10.1103/PhysRevLett.111.065503

PACS numbers: 61.72.Hh, 42.25.Kb, 61.05.cp, 62.20.fq

In the context of semiconductor device miniaturization down to the nanoscale, one of the most important challenges for the realization of highly efficient systems is to ensure a high crystalline quality of micro- or nanocrystals. Any deviation from the perfect atomic arrangement constitutes a "defect" [1] that can dramatically modify the device performance [2-4]. This is especially true for "phase" defects, characterized by a global shift of one part of the crystal with respect to another such as, for example, dislocations [5]. A technique that not only detects but also evaluates the number of defects in a small crystal is thus highly desirable for performing quantitative diagnostic and defect engineering studies. However, an efficient, fast, robust, and nondestructive defect characterization method has not been available previously. Transmission electron microscopy (TEM) can provide images of lattice defects, but this approach is limited to thin, electron transparent samples that often require a destructive sample preparation method. We demonstrate here that coherent $\mathrm{x}$ rays provide a very powerful method for detecting and quantifying small numbers of dislocations in microcrystals, without introducing any sample degradation. This is a significant step forward, compared to previous coherent $\mathrm{x}$-ray work, which has been limited to the study of single dislocations [6,7]. Our technique can be applied to a wide variety of systems and could become a standard characterization method which would be particularly valuable for nanotechnology.

A system for which the physical properties are modified by the introduction of dislocations is the III-V semiconductor compound InSb: brittle in the bulk state, it becomes ductile in microcrystal form [8]. This dramatic change in the mechanical properties is attributed to a change of deformation mechanism when reducing the crystal size: elastic strain accumulated in the crystal during mechanical loading is mostly released by cracks in bulk InSb, whereas this strain is dissipated via the formation of dislocations in small crystals [8]. Herein, InSb microcrystals are mechanically deformed up to the irreversible deformation limit (the elastic to plastic transition) to introduce a small number of controlled dislocations and subsequently study them with coherent $\mathrm{x}$ rays.

The sample is a $\operatorname{InSb}$ [213] single crystal, on which several InSb micropillars $(6 \mu \mathrm{m}$ in height, $2 \mu \mathrm{m}$ in diameter) have been machined using a focused ion beam (FIB). Each micropillar stands on a pedestal $16 \mu \mathrm{m}$ in diameter and $4 \mu \mathrm{m}$ high. Material surrounding the pedestal is removed by the FIB, forming a crater of radius $r_{c} \sim$ $80 \mu \mathrm{m}$ and leaving a free path for both incoming and diffracted x-ray beams (see Fig. 1). The bottom of the crater was observed to contain some clusters of amorphous $\mathrm{InSb}$ due to material redeposition during FIB machining.

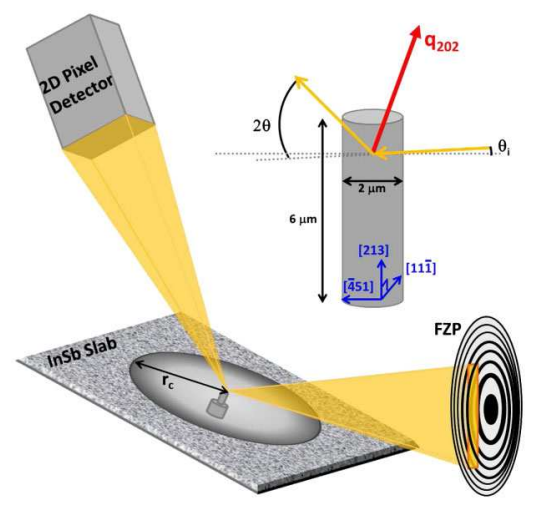

FIG. 1 (color online). Diffraction geometry. A coherent, slitselected beam illuminates the FZP and is focused on the chosen pillar. The diffracted beam is collected by a $2 \mathrm{D}$ pixel detector. The pillar stands on a pedestal and lies in a crater of radius $r_{c}$. Inset: Crystallographic directions, size of the pillar, and diffraction angles. 
Several pillars were deformed through application of a uniaxial compression using a flat tip indenter, up to the beginning of the plastic regime, i.e., after the first noticeable deviation from elasticity in the stress-strain curve [8]. Because of the specific [213] orientation of the pillars, only one defect type is created, with a single well-defined orientation (single slip orientation). These defects have been previously characterized by TEM and were found to be partial dislocations nucleating at the pillar surface [8]. Each leading partial dislocation glides through the pillar up to the opposite surface, leaving a stacking fault (SF) in the (111) plane. In the deformed pillar studied, a limited number of SFs is expected in parallel (11̄1) glide planes. The first SF always appears at the top edge of the pillar, and the subsequent faults are found below this.

The coherent diffraction experiment was performed at the ID01 beam line of the European Synchrotron Radiation Facility (ESRF). The sample was mounted on a pseudo 6-circles diffractometer specially designed for diffraction studies using submicrometer beams. A $7 \mathrm{keV}(\lambda=$ $1.771 \AA$ ) beam was selected with a channel-cut $\operatorname{Si}(111)$ monochromator providing a band pass $\Delta \lambda / \lambda=$ $1.4 \times 10^{-4}$. Fresnel zone plates (FZPs), $200 \mu \mathrm{m}$ in diameter and $70 \mathrm{~nm}$ in outermost zone width, were used to focus the X-ray beam to a size of $200(v) \times 500(h) \mathrm{nm}^{2}$ at sample position. A coherent beam was obtained by selecting a beam matching the transverse coherence lengths before the FZP [i.e., $60(v) \times 20(h) \mu \mathrm{m}^{2}$ ] to ensure a high degree of coherence and a reasonable flux on the sample [9-11]. This peculiar illumination of the FZP leads to an elongation of the focal depth up to a few millimeters [11]. A Maxipix pixel detector $\left(516 \times 516\right.$ square pixels, $55 \times 55 \mu \mathrm{m}^{2}$ pixel size) was used $1.19 \mathrm{~m}$ after the sample, providing a resolution in reciprocal space of $\delta q=1.49 \times 10^{-4} \AA^{-1}$. A schematic of the experimental geometry is shown in Fig. 1. The nonspecular 202 reflection was chosen for its convenient accessibility within a vertical scattering geometry. The [101] direction is tilted by $19.1^{\circ}$ from the direction of the sample normal vector [213], and the calculated Bragg angle at this x-ray energy is $2 \theta=45.48^{\circ}$. In order to access this reflection, the incident beam angle was $\theta_{i}=3.6^{\circ}$ with respect to the InSb slab surface. This asymmetric geometry, together with the limited $r_{c}$ due to FIB fabrication, imposed some restrictions on the reachable height $(h)$ from the top of the pillar. This height was easily obtained by geometrical considerations: $h=r_{c} \tan \theta_{i}=4 \mu \mathrm{m}$ (see Fig. 1).

A scanning x-ray diffraction microscopy method was used to locate the pillars on the substrate [12] and to record full 3D x-ray diffraction maps at different positions on the pillar. Spatial maps were recorded via scanning the sample laterally in two perpendicular directions, at different incidence angles around the 202 reflection. The lateral steps of these scans were 300 and $500 \mathrm{~nm}$ for vertical and horizontal directions, respectively. A step size of $0.01^{\circ}$ was utilized for angular scans.
The combination of spatial and angular scans allows for tracking of the pillar position at different incident angles and for correction of drifts. The stability is better than $50 \mathrm{~nm}$ for each spatial map. Analysis of images recorded at the same pillar position for different incidence angles through the 202 reflection allows for elucidation of the rocking curve at each pillar position. The measured full width at half maximum $\left(0.07^{\circ}\right)$ is limited by the vertical beam divergence and accounts for a small crystal mosaicity. A diffraction map of the pillar at the maximum of the 202 reflection is shown in Fig. 2(c). Three specific positions of the pillar are indicated and are analyzed in more detail. Attention is focused on the upper part of the pillar, known to be the initial defect formation location based on earlier scanning electron microscopy (SEM) studies [8].

To track possible phase defect effects on obtained diffraction patterns, in Fig. 2(a), we compare the images recorded at the rocking curve maximum for the three positions indexed in Fig. 2(c). The pixels of the detector have been converted into reciprocal space coordinates: $q_{x}$ is the vertical direction of the detector tilted by an angle $\theta$ with respect to the [202] direction, and $q_{y}$ is the horizontal direction of the detector along the [11ī] direction.

At the very top of the pillar (position 1), the peak is very sharp and appears at the calculated Bragg position. However, many speckles appear around it. At position 2 (1.2 $\mu \mathrm{m}$ from the top), the speckles have disappeared and the central maximum is split. At position $3(1.8 \mu \mathrm{m}$ from the top), the peak displays a single maximum again. The projections along the $q_{x}$ direction of the diffraction patterns recorded at positions 3 and 2 are displayed in Fig. 2(d).

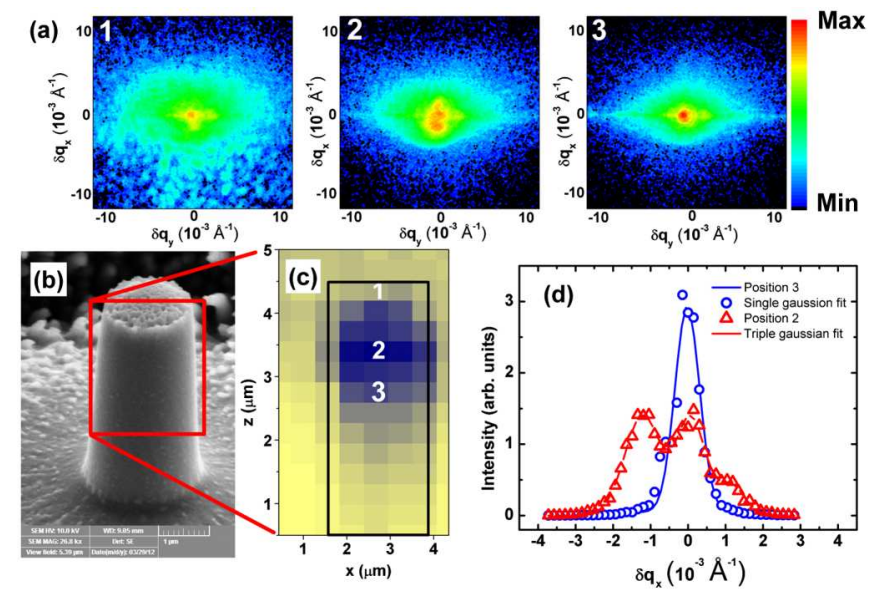

FIG. 2 (color online). (a) Coherent diffraction patterns obtained at the maximum of the 202 reflection at three positions indexed in (c) (log intensity scale). (b) SEM image of the studied deformed pillar. (c) Scanning diffraction image obtained at the maximum of the 202 reflection. (d) Projection along $q_{x}$ of the peaks obtained at positions 2 and 3, and fit with Gaussian functions. 
At position 1, the beam probes the top of the pillar. In this region, a single high intensity maximum is recorded in the center, indicating that the majority of the crystalline volume is perfect. Many low intensity speckles are recorded in the surrounding area and are attributed to small crystal degradation induced by FIB milling [8]. At position 3 , a single maximum also appears at the center of the diffraction pattern, but no speckles are recorded. The crystalline arrangement is perfect in this region. The most interesting region of the sample is located at position 2, where multiple peaks appear, although FIB-induced degradation is not observed [8]. The additional maxima at position 2 are clearly visible for projections along $q_{x}$ [Fig. 2(d)] and are attributed to the presence of phase defects introduced during plastic deformation. They appear along a line which is tilted by $\sim 20^{\circ}$ with respect to $q_{x}$ and whose direction is consistent with the projection of the [111] direction onto the detector plane. The multiple maxima cannot be attributed to tilted crystalline domains which would scatter at different angles along $q_{y}$. Moreover, for the projections shown in Fig. 2(d), the three Gaussian peaks have approximately the same width. This observation can only be explained as a result of interference effects between beams scattered by phase-shifted regions of the sample and separated by phase defects such as the SFs described earlier. These defects indeed lie in (1111) planes, as observed in TEM images [8].

A numerical study was then performed to investigate the effects of a limited number of SFs present in the probed volume. The presence of a SF introduces a phase shift of $2 \pi / 3$ in the (111) planes, resulting in a phase shift of $4 \pi / 3$ onto the (202) planes probed in our experiment. Calculations are made for SFs appearing in different fractions $(V)$ of the illuminated volume and for a different number of defects $(N)$. The initially perfect sample is modeled by a rectangular computing box (CB) of $26 \times$ 256 pixels of uniform density and phase, which describes the section of the pillar illuminated by the submicron $\mathrm{x}$-ray beam. The sample is then inserted in a larger numerical window $(260 \times 2048$ pixels $)$ to calculate the coherent diffraction patterns with a fast Fourier transform. In the sample CB, SFs are successively introduced as lines tilted by $45^{\circ}$ separating regions of different phases. This choice for the angle is driven by the need for isolation of the features related to the $\mathrm{CB}$ size or shape from the effects produced by the presence of SFs on the calculated reciprocal space image. The SFs are only inserted below the first one to mimic reality [see Fig. 3(a)]. This model provides an adequate description of the experimental conditions.

The case of a perfect crystal [Fig. 3(b)] is trivial: a perfect Bragg reflection with vertical and horizontal oscillations due to the finite CB size is obtained. When a single $\mathrm{SF}$ is introduced close to the center of the volume [Fig. 3(c)], a splitting appears, as well as a diffuse streak perpendicular to the SF plane. This situation has already
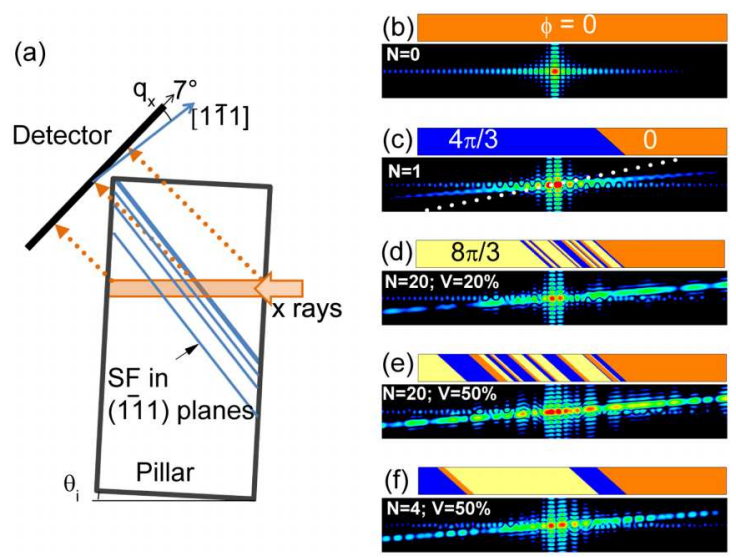

FIG. 3 (color online). (a) Sketch of the pillar probed by the beam at position 2. The blue lines denote SFs; the first that appears is above all others and is at a fixed position. The right part of the probed volume is always defect free. The vertical direction of the detector $\left(q_{x}\right)$ is tilted by $7^{\circ}$ with respect to the projection of the [1 11 ] direction onto the scattering plane. (b)-(f) Top rectangle: spatial phase configuration for a given number of defects $(N)$ included in a volume fraction $(V)$. Orange, blue, and yellow regions correspond to phases 0 , $4 \pi / 3$, and $8 \pi / 3$. Bottom rectangle: calculated diffraction pattern on the Bragg reflection (log scale) obtained by the fast Fourier transform of the spatial configuration. The horizontal and vertical scales are not normalized. The vertical direction of the detector $\left(q_{x}\right)$ cuts the diffraction pattern along the dotted white line shown in (c) and is tilted by $7^{\circ}$ with respect to the diffuse streak.

been reported experimentally for atomic [6] and electronic single dislocations $[13,14]$. The Bragg reflection is split due to the interference between the phase-shifted regions located on both sides of the defect, where each peak has the same width as the single peak measured in the case of the perfect crystal. However, in the case of several phase defects, the interferences measured in a coherent diffraction experiment are more difficult to interpret. Reference [15] shows how these complex patterns measured on misfit dislocations cannot be used for imaging with phase retrieval.

We next investigated the case of a constant number of SFs spread in different fractions of the illuminated volume. Configurations obtained for 20 SFs randomly spread in both $20 \%$ and $50 \%$ of the total illuminated volume are shown in Figs. 3(d) and 3(e), with corresponding calculated coherent diffraction patterns. When 20 SFs are confined in $20 \%$ of the illuminated volume, the diffraction pattern is very similar to the case of a single SF. A split reflection only appears provided that the volumes on both sides of the defected area are phase shifted, and the streak perpendicular to the SF direction displays oscillations with a period which is inversely proportional to the size of the defected volume. In this case, the intensity distribution is dominated by the diffraction from two phase-shifted volumes on the two sides of the defected volume. When 20 


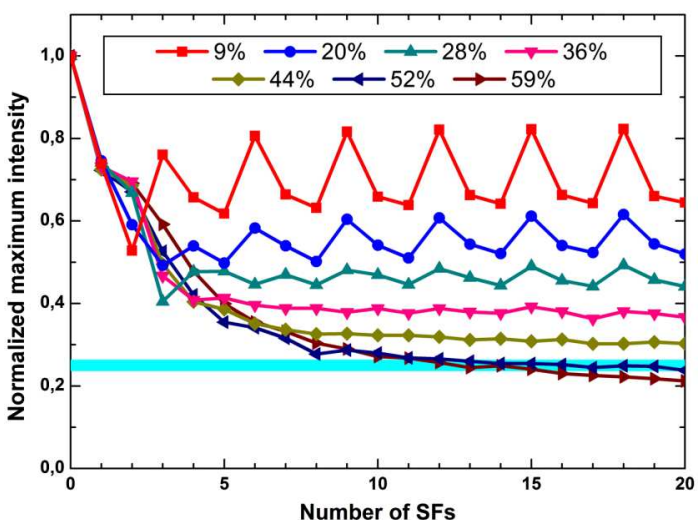

FIG. 4 (color online). Evolution of the maximum intensity obtained on the diffraction patterns for increasing number of SFs, for different defected volume sizes. The blue line represents the ratio between the maximum intensities recorded at positions 2 and 3 during the experiment.

SFs are randomly inserted in a fraction of $V=50 \%$, the diffraction pattern contains many speckles aligned along a direction perpendicular to the SFs. The interference peaks located around the Bragg position are more intense than the others, but their arrangement along the intensity "streak" highly depends on the spatial configuration of the SFs. This is an analogous behavior to SFs observed in nanowires, in which wurtzite and zinc-blende domains are alternatively found [16]. In this case, it is not trivial to retrieve the real space configuration of the SFs from the recorded diffraction patterns.

The influence of the number of defects $(N)$ is also of high importance. When defects are increased from $N=4$ to $N=20$ at a constant fraction of $V=50 \%$ [Figs. 3(e) and 3(f)], the number of speckles on the corresponding diffraction patterns also increases and the streak is structured accordingly.

Comparing these numerical simulations to our experimental findings, we see that if one only considers the diffraction pattern image, many spatial configurations could lead to the observed patterns. This finding is in agreement with reports found in the literature. However, we observe that the maximum intensity obtained in the diffraction patterns for different configurations holds great information. The maximum intensity strongly depends on the number of defects and on the fraction of illuminated volume in which they appear. The evolution of the calculated maximum intensity as a function of the number of SFs is displayed in Fig. 4 for different fractions of defected volume. For each point on these curves, an average over 200 random configurations was performed. As the volume fraction is only meaningful for $N \geq 2$, the positions of the two first SFs are fixed (the first SF invariably at the same upper position). Only the subsequent SFs are inserted at a random position in this volume, without superimposition. The intensity maximum on the calculated diffraction pattern is then normalized with respect to the one obtained with no SF. The statistical relative error in the calculation is always lower than $10^{-3}$.

For SFs appearing in large volume fractions, the maximum intensity decays rapidly with an increasing number of defects. For smaller defected volumes, the maximum intensity also decays with the number of defects, but the saturation regime is obtained earlier and the saturation level higher. For very small fractions $(V \leq 30 \%)$, the maximum intensity is higher for $N=3 n, n \in \mathbb{N}$. This situation indeed leads to in-phase volumes on both sides of the defected region, so that a single maximum is found in the center of the pattern. In our case, the ratio between the maximum intensities found experimentally at the defected and perfect positions (respectively, positions 2 and 3) is $0.25 \pm 0.01$ (Fig. 4). Thus, $\sim 15$ defects are illuminated at position 2, in a fraction $V \sim 50 \%$. Further, 15 partial dislocations (nucleated in individual slip planes) induce an axial pillar deformation of $\sim 3 \mathrm{~nm}$ and correspond to a strain of $\sim 0.05 \%$. As expected, such a value corresponds to the early plastic regime (below $0.2 \%$ ) and to a still detectable deviation in the stress-strain curve. However, such a small number of fine slip traces at the pillar's surface can hardly be seen using SEM. This demonstrates the very high sensitivity of coherent $\mathrm{x}$ rays, increasingly apparent for a small number of defects (see Fig. 4).

The results presented in this Letter are highly promising for the study of small-scale materials with defectdependent physical properties. We believe this method will become a valuable diagnostic and quality assessment tool for nanofabrication processes and allow for the in situ monitoring of defect nucleation in defect-free nanostructures. Moreover, within a defect engineering approach, it should provide answers to fundamental questions, such as how and when the first defects appear in microcrystals (under different external stimuli) or how many and which defects affect the micro-object properties.

The authors are grateful to the beam line scientist $\mathrm{T}$. Schülli for his support and to $\mathrm{H}$. Djazouli for technical assistance during the experiment.

*vincent.jacques@u-psud.fr

[1] J.P. Hirth and J. Lothe, Theory of Dislocations (McGraw-Hill, New York, 1968).

[2] F. Bonell, S. Andrieu, C. Tiusan, F. Montaigne, E. Snoeck, B. Belhadji, L. Calmels, F. Bertran, P. Le Fèvre, and A. Taleb-Ibrahimi, Phys. Rev. B 82, 092405 (2010).

[3] B. M. Moskowitz, J. Geophys. Res. 98, 18011 (1993).

[4] J. Kundu, C. Sarkar, and P. Mallick, Semi. Phys. Quant. Electron. Optoelectron. 10, 1 (2007).

[5] L. M. Giovane, H.-C. Luan, A. M. Agarwal, and L. C. Kimerling, Appl. Phys. Lett. 78, 541 (2001).

[6] V. L. R. Jacques, S. Ravy, D. Le Bolloc'h, E. Pinsolle, M. Sauvage-Simkin, and F. Livet, Phys. Rev. Lett. 106, 065502 (2011). 
[7] Y. Takahashi, A. Suzuki, S. Furutaku, K. Yamauchi, Y. Kohmura, and T. Ishikawa, Phys. Rev. B 87, 121201 (2013).

[8] L. Thilly, R. Ghisleni, C. Swistak, and J. Michler, Philos. Mag. 92, 3315 (2012).

[9] A. Diaz, C. Mocuta, J. Stangl, M. Keplinger, T. Weitkamp, F. Pfeiffer, C. David, T.H. Metzger, and G. Bauer, J. Synchrotron Radiat. 17, 299 (2010).

[10] V. L. R. Jacques, D. Le Bolloc'h, E. Pinsolle, F.-E. Picca, and S. Ravy, Phys. Rev. B 86, 144117 (2012).

[11] F. Mastropietro, D. Carbone, A. Diaz, J. Eymery, A. Sentenac, T. H. Metzger, V. Chamard, and V. FavreNicolin, Opt. Express 19, 19223 (2011).
[12] C. Mocuta, J. Stangl, K. Mundboth, T. Metzger, G. Bauer, I. Vartanyants, M. Schmidbauer, and T. Boeck, Phys. Rev. B 77, 245425 (2008).

[13] V.L.R. Jacques, D. Le Bolloc'h, S. Ravy, C. Giles, F. Livet, and S. B. Wilkins, Eur. Phys. J. B 70, 317 (2009).

[14] D. LeBolloc'h, S. Ravy, J. Dumas, J. Marcus, F. Livet, C. Detlefs, F. Yakhou, and L. Paolasini, Phys. Rev. Lett. 95, 116401 (2005).

[15] I. Robinson, Y. Da, T. Spila, and J. E. Greene, J. Phys. D 38, A7 (2005).

[16] V. Favre-Nicolin et al., New J. Phys. 12, 035013 (2010). 